\title{
Das Funktionale Basismodell für die gemeindepsychiatrische Versorgung schwer psychisch kranker Menschen - Mindest- standard für Behandlung und Teilhabe
}

\author{
The Functional Basic Model for the Psychiatric Care of Persons with Severe \\ Mental Illness - Minimum Standard of Treatment and Participation
}

Autoren

Institute
Ingmar Steinhart ${ }^{1}$, Günther Wienberg ${ }^{2}$

Institut für Sozialpsychiatrie Mecklenburg-Vorpommern, Ernst-Moritz-Arndt-Universität Greifswald

${ }^{2}$ Fachbereich Sozialwesen, Hochschule Fulda

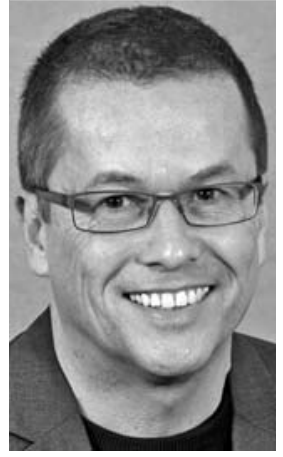

Ingmar Steinhart

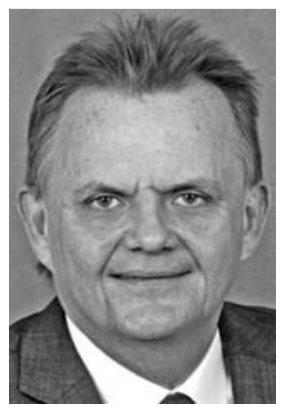

Günther Wienberg

Bibliografie

DOI http://dx.doi.org/ 10.1055/s-0042-101534

Psychiat Prax 2016; 43: 65-68

(c) Georg Thieme Verlag KG

Stuttgart · New York

ISSN 0303-4259

\section{Korrespondenzadresse}

Prof. Dr. Ingmar Steinhart Institut für Sozialpsychiatrie Mecklenburg-Vorpommern, An-Institut der Ernst-MoritzArndt-Universität Greifswald Ellernholzstraße 1-2 17487 Greifswald

institut@sozialpsychiatrie-mv.de
Zwischen 1 und 2\% der deutschen Erwachsenenbevölkerung oder 500000 bis 1 Mio. Menschen leiden zu jedem Zeitpunkt an einer schweren psychischen Erkrankung [1]. Auch gut 40 Jahre nach Vorlage der Psychiatrie-Enquete bestehen in Deutschland nach wie vor erhebliche Defizite in der Versorgung dieser Personengruppe [2]: Schwer psychisch kranke Menschen können zeitweise oder sogar auf Dauer vom vertragsärztlichen System nicht ausreichend profitieren, u.a. weil sie die Regelangebote der niedergelassenen Fachärzte und Psychotherapeuten nicht zuverlässig wahrnehmen können und weil dort die Ressourcen für ihre aufwendigere Behandlung fehlen. Außerdem sind sie regelhaft und erheblich von sozialer Exklusion bedroht. Die bedarfsgerechte Behandlung und Gewährleistung umfassender Teilhabechancen für diese Personengruppe ist gegenwärtig die zentrale Herausforderung des psychiatrisch-psychotherapeutischen Versorgungssystems.

Die systembezogenen Empfehlungen der S3-Leitlinie „Psychosoziale Therapien bei schweren psychischen Erkrankungen“ [3] stellen ambulante mobile multiprofessionelle gemeindepsychiatrische Teams in das Zentrum der Versorgung. Auch die UN-Konvention über die Rechte von Menschen mit Behinderungen (UN-BRK) definiert klare Standards. Dies gilt für allgemeine Grundsätze wie Würde, Autonomie, Freiheit, Nichtdiskriminierung, volle und wirksame Teilhabe, Chancengleichheit und Zugänglichkeit (Art.3) sowie für spezielle Rechte, wie die auf Gesundheitsversorgung (Art.25), Arbeit (Art.27) sowie umfassende Habilitation und Rehabilitation (Art.26). Hervorzuheben ist insbesondere, das Recht auf Unabhängige Lebensführung (Art.19), verbunden mit Wahlmöglichkeiten wo und mit wem man leben möchte, dem Zugang zu gemeindenahen Unterstützungsdiensten auch zu Hause und der persönlichen Assistenz zur Unterstützung des Lebens in der Gemeinschaft.
Die gegenwärtige versorgungspolitische Diskussion in Deutschland ist stark auf das Vergütungssystem PEPP für die (teil)stationäre Psychiatrie und Psychosomatik konzentriert, welche das zentrale Thema der Stärkung der ambulanten Versorgung weitgehend ausklammert. Dies gilt auch für ein Alternativmodell der Fachgesellschaften und Verbände vom September 2015 [4]. Auf der anderen Seite haben sich in den letzten Jahren in vielen Regionen sektorübergreifende Versorgungsmodelle mit dem Ziel etabliert, die ambulante Versorgung zu stärken. Dabei handelt es sich um Modellprojekte nach §64b SGB V, im Wesentlichen regionale Krankenhausbudgets $[5,6]$, um Modelle der Integrierten Versorgung nach $§ 140$ a-d SGB V oder um innovative Modelle im Rahmen der Regelfinanzierung nach $\S 118$ SGB V oder nach $\S 17 \mathrm{~d}$ KHG [7].

Die Grenzen der regionalen Krankenhausbudgets liegen vor allem darin, dass sie andere Leistungssegmente bzw. -erbringer nach dem SGB V außen vor lassen und das Leistungsgeschehen für die Leistungsträger intransparent ist. IV-Modelle kranken daran, dass vielfach der Personenkreis schwer psychisch kranker Menschen entweder direkt oder über die Einschreibehürde der Selektivverträge ausgeschlossen wird. Sie basieren darüber hinaus zumeist auf kassenspezifischen Verträgen und tragen so zu einer weiteren Zersplitterung der Versorgungslandschaft bei. Fast allen Modellen ist gemeinsam, dass sie den Bereich der Teilhabe außen vor lassen, das heißt insbesondere Leistungen nach den SGB IX und XII. Dabei steht gerade mit dem Bundesteilhabegesetz (BTG) in 2017ff. eine weitreichende Neuausrichtung der Eingliederungshilfe in Aussicht, u.a. mit der Einführung einer Beratungsleistung, der Stärkung der Personenorientierung und der ambulanten Leistungserbringung.

Vor diesem Hintergrund haben die Autoren dieses Beitrags in einem früheren Editorial dieser Zeitschrift [8] ein funktionales Basismodell für die ge- 


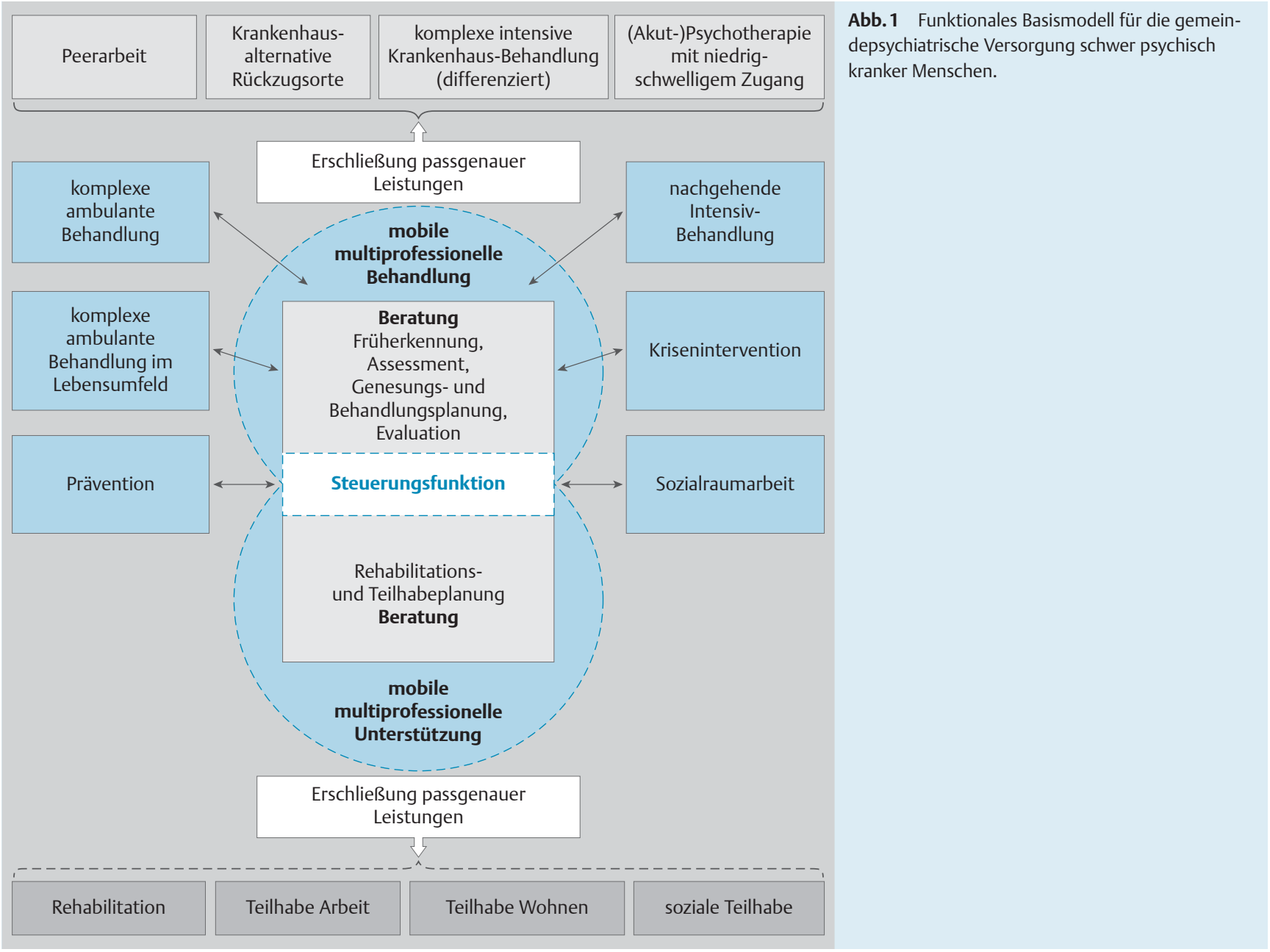

meindepsychiatrische Versorgung schwer psychisch kranker Menschen vorgeschlagen. Dieses Modell basiert auf den Empfehlungen der S3-Leitlinie und definiert mobile multiprofessionelle Teams als Zentrum der Behandlung, von der aus bei Bedarf weitere ergänzende ambulante und (teil)stationäre Leistungen erschlossen werden. Die erste Fassung des Modells beschränkte sich noch auf Leistungen der gesetzlichen Krankenversicherung (GKV), auf die Notwendigkeit der Einbindung weiterer Leistungen außerhalb des SGB V wurde lediglich verwiesen. Es war als Diskussionsvorschlag gedacht und ist inzwischen mit zahlreichen Fachkolleginnen und -kollegen diskutiert worden. Die Rückmeldungen und Anregungen waren Ermutigung, das Modell um den Bereich der Teilhabe zu erweitern [9] und hier eine erneut überarbeitete Fassung ( $\bullet$ Abb. 1) vorzulegen.

Die Kernfunktion mobile multiprofessionelle Behandlung (einschließlich Beratung, Früherkennung, Assessment, Genesungsbzw. Behandlungsplanung und Evaluation) wird dabei verknüpft mit der Kernfunktion mobile multiprofessionelle psychosoziale Unterstützung (einschließlich Beratung sowie Rehabilitationsund Teilhabeplanung). Beide Kernfunktionen integrieren die Leistungen personenbezogen, stellen die konzeptionelle und personelle Kontinuität sicher, erschließen bei Bedarf passgenau ergänzende Leistungen und üben damit eine zentrale Steuerungsfunktion aus. Zum Leistungsspektrum der mobilen multiprofessionellen Dienste gehörten auch fallunspezifische Leistungen wie Gesundheitsförderung und Prävention sowie Netzwerkarbeit im Sozialraum. Das Modell denkt Behandlung und Unterstützung zur Teilhabe konsequent aus der ambulanten Perspektive, weitere - auch (teil)stationäre - Leistungen werden als Ergänzung betrachtet. Es beansprucht damit, einen Mindeststandard für die bedarfsgerechte Versorgung von Menschen mit schweren psychischen Erkrankungen in der Gemeinde zu definieren. Das heißt, die aufgeführten Funktionen sind in jeder Versorgungsregion obligatorisch sicherzustellen, unabhängig von ihrer organisatorischen Umsetzung.

Dabei kann und muss auf die jeweils regional vorhandenen Ressourcen zurückgegriffen werden. Denn eine On-top-Finanzierung, insbesondere der mobilen multiprofessionellen Teams, wäre ökonomisch kaum durchsetzbar und fachlich kontraproduktiv, da sie neue Parallelstrukturen schaffen würde. In $\bullet$ Tab. 1 werden die Funktionen des Basismodells in Beziehung gesetzt zu (potenziellen) Ressourcen in einer Versorgungsregion, die diese Funktionen ausfüllen können. Dabei kann es sich um Dienste und Einrichtungen, Personen(-Gruppen) und Technologien handeln. Letzteres mag überraschen, aber nicht zuletzt die UN-BRK postuliert den ungehinderten Zugang von Menschen mit Behinderungen zu Informations-, Kommunikations- und unterstützenden Technologien (vgl. Art. 4 und 7). Bei der Zusammenschau der Ressourcen wurde auf aktuelle Übersichtsarbeiten und Datenbestände zurückgegriffen [7-11]. Sie fokussiert den Bereich der Allgemeinpsychiatrie, Erweiterungen auf Gerontopsychiatrie und Suchthilfe sind unschwer möglich.

Mit Blick auf die Kernfunktionen der multiprofessionellen mobilen ambulanten Behandlung und psychosozialen Unterstützung 
Tab. 1 Basisfunktionen und Ressourcen in der gemeindepsychiatrischen Versorgung schwer psychisch kranker Menschen.

\begin{tabular}{|c|c|c|c|}
\hline & & Ressourcen & \\
\hline Funktionen & $\begin{array}{l}\text { in zahlreichen Regionen } \\
\text { bis flächendeckend verfügbar }\end{array}$ & $\begin{array}{l}\text { in einer größeren Zahl von } \\
\text { Regionen verfügbar, gut erprobt }\end{array}$ & $\begin{array}{l}\text { nicht bis nur vereinzelt } \\
\text { verfügbar | Modellprojekte }\end{array}$ \\
\hline Peer-Arbeit & Peer-Selbsthilfe & Genesungsbegleiter, Peer-Berater & \\
\hline $\begin{array}{l}\text { Krankenhausalternative } \\
\text { Rückzugsorte }\end{array}$ & & $\begin{array}{l}\text { Krisenwohnungen/-pensionen, } \\
\text { Soteria-Milieus }\end{array}$ & Gastfamilien \\
\hline Krankenhausbehandlung & $\begin{array}{l}\text { geschlossene, offene, ggf. speziali- } \\
\text { sierte Krankenhausstationen, } \\
\text { Tageskliniken }\end{array}$ & & $\begin{array}{l}\text { 7-Tage-Tageskliniken, } \\
\text { Halb-Tageskliniken }\end{array}$ \\
\hline Akut-Psychotherapie, niedrigschwellig & & PIA & $\begin{array}{l}\text { niedergelassene Psychothera- } \\
\text { peuten }\end{array}$ \\
\hline $\begin{array}{l}\text { Komplexe ambulante Behandlung } \\
\text { bei Bedarf im Lebensumfeld } \\
\text { bei Bedarf nachgehend intensiv } \\
\text { mit Krisenintervention } 7 \text { T./24 Std. }\end{array}$ & $\begin{array}{l}\text { PIA } \\
\text { Sozialpsychiatrische Dienste }\end{array}$ & $\begin{array}{l}\text { ambulante psychiatrische Pflege } \\
\text { ambulante Soziotherapie } \\
\text { psychosoziale Beratungsstellen }\end{array}$ & $\begin{array}{l}\text { spezielle Krisendienste, nieder- } \\
\text { gelassene Fachärzte } \\
\text { niedergelassene Psychothera- } \\
\text { peuten }\end{array}$ \\
\hline Berufliche Rehabilitation & $\begin{array}{l}\text { RPK, IFD, AET, BBW/BFW, BTZ, } \\
\text { WfbM }\end{array}$ & $\begin{array}{l}\text { unterstützte Beschäftigung } \\
\text { (befristet) }\end{array}$ & $\begin{array}{l}\text { PIA ggf. plus spezialisierte } \\
\text { (Sozial)Dienste der Kliniken }\end{array}$ \\
\hline Teilhabe am Arbeitsleben & $\begin{array}{l}\text { WfbM, } \\
\text { Tagesgestaltung mit Arbeits- } \\
\text { charakter }\end{array}$ & $\begin{array}{l}\text { Integrationsfirmen } \\
\text { Zuverdienst }\end{array}$ & $\begin{array}{l}\text { UA auf dem allgemeinen Arbeits } \\
\text { markt (unbefristet) }\end{array}$ \\
\hline Teilhabe im Wohnbereich & $\begin{array}{l}\text { UW in eigener Wohnung, in einer } \\
\text { Wohngruppe, in institutionellem } \\
\text { Setting (bei Bedarf geschlossen) }\end{array}$ & $\begin{array}{l}\text { UW in einer Gastfamilie, } \\
\text { IAUW }\end{array}$ & $\begin{array}{l}\text { technische Assistenzsysteme zur } \\
\text { Unterstützung selbstständigen } \\
\text { Wohnens }\end{array}$ \\
\hline soziale Teilhabe & $\begin{array}{l}\text { Tagesstätten, } \\
\text { Kontakt-/Begegnungsstätten, } \\
\text { Selbsthilfegruppen }\end{array}$ & & \\
\hline
\end{tabular}

AET = Ambulante Ergotherapie, BBW = Berufsbildungswerk, BFW= Berufsförderungswerk, BTZ= Berufliches Trainingszentrum; IAUW=Intensiv ambulant unterstütztes Wohnen, IFD = Integrationsfachdienst, PIA = Psychiatrische Institutsambulanz, RPK = Rehabilitationseinrichtung für psychisch Kranke. WfbM=Werkstatt für behinderte Menschen, $\mathrm{UA}=$ Unterstütztes Arbeiten, UW= Unterstütztes Wohnen.

wird deutlich, dass PIA und Sozialpsychiatrische Dienste eine flächendeckend verfügbare Ressource darstellen, auf die nicht verzichtet werden kann. Aber auch niedergelassene Fachärzte in Kooperation mit ambulanter psychiatrischer Pflege, Soziotherapie und ambulanter Psychotherapie stellen wichtige potenzielle Ressourcen dar. Bei den ergänzenden Behandlungsfunktionen (obere Hälfte - Tab.1) wird deutlich, dass die entsprechenden Ressourcen weitgehend krankenhausgebunden sind, die Peer-Arbeit durch Genesungsbegleiter (Beteiligung an professioneller Hilfe) und Peer-Berater ist ebenso wenig flächendeckend verfügbar wie krankenhausalternative Milieus. Die Funktion niedrigschwellige Akut-Psychotherapie wird gegenwärtig allenfalls von einer Teilgruppe der PIA wahrgenommen, die anstehende Novellierung des Psychotherapeutengesetzes könnte hier eine Chance zur Verbesserung bringen.

Im Bereich der Leistungen zur Teilhabe am Arbeitsleben sind Angebote der beruflichen Rehabilitation recht weit, wenn auch nicht flächendeckend verbreitet. Nicht alle diese Angebote werden jedoch den besonderen Bedarfen und Bedürfnissen schwer psychisch kranker Menschen gerecht. Insbesondere Berufsbildungswerke (BBW) und Berufsförderwerke (BFW) sind in der Regel gemeindeferne, stationäre Großeinrichtungen. Fast völlig fehlen in Deutschland Angebote des unbefristet unterstützten Arbeitens, eine Funktion, für deren Wirksamkeit es eine breite, internationale wissenschaftliche Evidenz gibt [12]. Das Standardangebot für unbefristete Arbeit sind immer noch die WfbM mit anhaltend hohen Zuwachsraten für schwer psychisch kranke Menschen [13]. Bezüglich der Teilhabe im Bereich Wohnen gibt es in Deutschland ein flächendeckendes Netz von Wohnheimen sowie an Diensten des unterstützten Wohnens (zumeist als betreutes Wohnen bezeichnet). Weniger verbreitet ist das unterstützte
Wohnen in Gastfamilien sowie das intensiv ambulant unterstützte Wohnen. Hierzu gibt es z.B. in Westfalen ein Modell, in dem bei festgestelltem stationären Unterstützungsbedarf dem Wunsch des Klienten nach intensiver ambulanter Unterstützung entsprochen wird mit einem Budget, das nur wenig unter dem Heim-Entgeltsatz liegt [14]. Technische Unterstützungs- und Kommunikationssysteme für ein möglichst langes selbstständiges Leben in der eigenen Wohnung sind im Hinblick auf ihre Bedeutung für Menschen mit psychischen Erkrankungen bisher kaum entwickelt und erprobt.

Das Basismodell formuliert einerseits Mindeststandards zur Sicherstellung der Funktionen in einer Versorgungsregion, andererseits enthält es nur wenige Festlegungen im Hinblick auf die strukturelle und organisatorische Umsetzung. Es ist also für jede Region auf Basis ihrer Ressourcen zu klären, welche Akteure mit welchen Leistungsangeboten bereit und in der Lage sind und welche Organisationsform geeignet ist, die Funktionalitäten des Basismodells zu realisieren. Dabei geht es jedoch um mehr als um eine bloße Addition bestehender Dienste und Einrichtungen sowie deren forcierte „Ambulantisierung“. Die Kernfunktionen „Steuerung“ und „Mobile multiprofessionelle Behandlung/Unterstützung“ machen eine rechtsfähige Organisationsform erforderlich, wenn sie wirksam sein sollen. Denn bei der hier intendierten Steuerung des Leistungsgeschehens geht es um eine erhebliche Ressourcenverschiebung aus dem stationären in den ambulanten Sektor. Ohne die regionalen Anbieter der stationären psychiatrischen Pflichtversorgung und ohne die Ressourcen (sprich: Plätze) der Heim- und Werkstattanbieter, den damit verbundenen Budgets und ihrem Willen, alle Funktionen mit ambulantem Kern umzusetzen, wird es nicht gehen. Eine Umgehung der stationären Anbieter und damit der Aufbau von Parallelstrukturen 
sind unrealistisch, weil nicht finanzierbar. Potente Anbieter stationärer Leistungen werden sich allerdings auf derartige Prozesse nur einlassen, wenn sie entsprechende Deckungsbeiträge auch mit ambulanten Leistungen generieren können.

Aus fachlich-organisatorischer Sicht wird die Umstellung auf das Basismodell für alle beteiligten Akteure gravierend sein, weil es nicht nur eine personenzentrierte Vorgehensweise, sondern vor allem eine primär nicht institutionelle und damit lebensweltbezogene Leistungserbringung erfordert. Einige Funktionen wie Prävention und Sozialraumarbeit müssen - auch das ist ungewohnt - ohne Bezug zu einem Einzelfall umgesetzt werden. Auch die Einbindung der Kompetenz von Psychiatrieerfahrenen in Form von Peer-Beratern oder Genesungsbegleitern erfordert neue und kreative Umsetzungsformen. Als regionale Organisationsform hat die Expertenkommission von 1988 den Gemeindepsychiatrischen Verbund (GPV) als Standardlösung vorgeschlagen. Die bundesweiten Erfahrungen damit zeigen bei aller Unterschiedlichkeit der regionalen Ausgestaltung, dass diese - abgesehen von wenigen Ausnahmen - bisher kaum über eine Bündelung der Anbieterinteressen hinausgekommen sind und nur in wenigen Fällen eine Neuausrichtung der Leistungsangebote bewirkt haben. Ein Abgleich mit den Anforderungen des Funktionalen Basismodells würde ein ernüchterndes Bild abgeben. Die zur Umsetzung des Basismodells in den meisten Regionen erforderliche Umgestaltung der Versorgungslandschaft dürfte ausschließlich in Kooperationsbezügen ohne eine entsprechende Rechtsform nicht erreichbar sein. Zu klären ist hier auch, ob die Gebietskörperschaften willens und fachlich wie personell in der Lage sind, die Federführung bei einem solchen Umbauprozess $\mathrm{zu}$ übernehmen und wie die Beteiligung Psychiatrieerfahrener in entsprechenden Prozessen gewährleistet werden kann.

Auch die Finanzierung eines solchen ambulant gestützten, funktional organisierten gemeindepsychiatrischen Systems muss neu angelegt werden, nämlich sektor- und SGB-übergreifend, ein Novum für die deutsche Versorgungslandschaft (ein Modellprojekt hat im letzten Jahr im Kreis Dithmarschen begonnen [15]).

Es gilt also zusammengefasst,

- die Entwicklung der Angebotsstrukturen vor Ort an den Mindeststandards des funktionalen Basismodells auszurichten

- SGB-übergreifende Organisations- und Finanzierungsmodelle zu generieren und

- das GPV-Modell in rechtsfähige Strukturen zu überführen bzw. eine Alternativlösung mit „starker“ Steuerungsfunktion zu entwickeln.

Für diese 3 essenziellen Anforderungen sollten 40 Jahre nach der Psychiatrie-Enquete im Rahmen von Modellprojekten, ggf. finanziert über den Innovationsfond, nach innovativen Lösungen gesucht werden.

\section{Literatur}

1 Gühne U, Becker T, Salize HJ et al. Wie viele Menschen in Deutschland sind schwer psychisch krank? Psychiat Prax 2015; 42: 415-423

2 Wienberg G. 40 Jahre Psychiatriereform in Deutschland - Auf dem Weg in die Drei-Klassen-Psychiatrie? Sozialpsychiat Inform 2013; 43: 1-9

3 Deutsche Gesellschaft für Psychiatrie und Psychotherapie, Psychosomatik und Nervenheilkund (DGPPN). S3-Leitlinie Psychosoziale Therapien bei schweren psychischen Erkrankungen. Berlin: Springer; 2013

4 Fachgesellschaften und Verbände der Plattform Entgelt. Konzept für ein Budgetbasiertes Entgeltsystem. Im Internet: https://www.dgppn. de/fileadmin/user_upload/medien/download/pdf/stellungnahmen/ 2015/2015-09-09_Plattf (Stand: 10.1.2016)

5 Deister A, Wilms B. Regionale Verantwortung übernehmen - Modellprojekte nach § 64b SGB. Köln: Psychiatrie-Verlag; 2014

6 Schmid P, Steinert T, Borbé R. Systematische Literaturübersicht zur Implementierung der sektorübergreifenden Versorgung (Regionalbudget, integrierte Versorgung) in Deutschland. Psychiat Prax 2013; 40: $414-424$

7 Steinhart I, Wienberg G, Koch C. Krankenhausersetzende psychiatrische Behandlung in Deutschland - Praxismodelle, Standards und Finanzierung. GGW 2014; 14: $15-26$

8 Steinhart I, Wienberg G. Plädoyer für ein funktionales Basismodell gemeindepsychiatrischer Versorgung. Psychiat Prax 2014; 41: 179-181

9 Steinhart I, Wienberg G. Mindeststandards für Behandlung und Teilhabe - Plädoyer für ein funktionales Basismodell gemeindepsychiatrischer Versorgung schwer psychisch kranker Menschen. Sozialpsychiat Inform 2015; 45: 9-15

10 Stengler K, Riedel-Heller S, Gühne U et al. Gemeindepsychiatrische Versorgung. Psychup2date 2015; 9: 113-128

11 Arbeitsgemeinschaft der Obersten Landesgesundheitsbehörden (AOLG). Tabellenanhang zum Bericht „Psychiatrie in Deutschland Strukturen, Leistungen, Perspektiven an die Gesundheitsministerkonferenz 2012. Im Internet: https://www.gesunde.sachsen.de/down load/Download_Gesundheit/Anlagen_GMK-Bericht_2012_der_AG_ Psychiatrie_der_AOLG.pdf (Stand 10.1.2016)

12 Gühne $U$, Riedel-Heller S. Die Arbeitssituation von Menschen mit schweren psychischen Erkrankungen in Deutschland. Im Auftrag von Gesundheitsstadt Berlin und DGPPN. Im Internet: https://www. dgppn.de/fileadmin/user_upload/_medien/dokumente/schwerpunk te/Expertise_Arbeitssituation_2015-09-14_fin.pdf (Stand: 10.1.2016)

13 Bundesarbeitsgemeinschaft der überörtlichen Sozialhilfeträger (BAGüS), consens. Kennzahlenvergleich der Überörtlichen Träger der Sozialhilfe 2012. Im Internet: https://www.consens-info.de/upload/ files/projekte/z_projekte_dateien_118704_ueoeTr_Kennzahlenver gleich_2012_Endfassung_31_01_14.pdf (Stand: 10.1.2016)

14 Steinhart I. Mietvertrag statt Heimvertrag. Lösungen zur Umsetzung der UN-Behindertenrechtskonvention. Sozialpsychiat Inform 2014; 45: $14-18$

15 Faulbaum-Decke $W$, Hohage $R$. Eingliederungshilfe im gegliederten System der sozialen Sicherung. Kerbe 2015; 4: 40-43 